\title{
Selection for uniformity in livestock by exploiting genetic heterogeneity of residual variance
}

\author{
Han A. MULDER ${ }^{1 *}$, Piter BIJMA ${ }^{1}$, William G. HILL ${ }^{2}$ \\ ${ }^{1}$ Animal Breeding and Genomics Centre, Wageningen University, $6700 \mathrm{AH}$ Wageningen, \\ The Netherlands \\ ${ }^{2}$ Institute of Evolutionary Biology, School of Biological Sciences, University of Edinburgh, \\ Edinburgh, EH9 3JT, UK
}

(Received 30 January 2007; accepted 23 August 2007)

\begin{abstract}
In some situations, it is worthwhile to change not only the mean, but also the variability of traits by selection. Genetic variation in residual variance may be utilised to improve uniformity in livestock populations by selection. The objective was to investigate the effects of genetic parameters, breeding goal, number of progeny per sire and breeding scheme on selection responses in mean and variance when applying index selection. Genetic parameters were obtained from the literature. Economic values for the mean and variance were derived for some standard non-linear profit equations, e.g. for traits with an intermediate optimum. The economic value of variance was in most situations negative, indicating that selection for reduced variance increases profit. Predicted responses in residual variance after one generation of selection were large, in some cases when the number of progeny per sire was at least 50, by more than $10 \%$ of the current residual variance. Progeny testing schemes were more efficient than sib-testing schemes in decreasing residual variance. With optimum traits, selection pressure shifts gradually from the mean to the variance when approaching the optimum. Genetic improvement of uniformity is particularly interesting for traits where the current population mean is near an intermediate optimum.
\end{abstract}

heterogeneity of variance / index selection / uniformity / economic value / optimum trait

\section{INTRODUCTION}

Uniformity of livestock is of economic interest in many cases. For example, the preference for some meat quality traits, such as $\mathrm{pH}$, is to be in a narrow range [19]. Farmers get premiums when they deliver animals in the preferred range and penalties for animals outside it [20]. Uniformity of animals and animal products is also of interest for traits with an intermediate optimum value,

\footnotetext{
*Corresponding author: herman.mulder@wur.nl
} 
such as litter size in sheep [37], egg weight in laying hens [10], carcass weight and carcass quality traits in pigs and broilers [11,14,19], marbling in beef [1]. Different strategies can be used to reduce variability, e.g. management, mating systems and genetic selection [18], but selection can be effective only when genetic differences in phenotypic variability exist among animals.

There is some empirical evidence for the presence of genetic heterogeneity of residual variance, meaning that genotypes differ genetically in phenotypic variance. San Cristobal-Gaudy et al. [37], in the analysis of litter size in sheep, and Sorensen and Waagepetersen [38], in the analysis of litter size in pigs, found substantial genetic heterogeneity of residual variance. Van Vleck [39] and Clay et al. [7], in the analysis of milk yield in dairy cattle, and Rowe et al. [35], in the analysis of body weight in broiler chickens, found large differences between sires in phenotypic variance within progeny groups. In these studies, heritabilities of residual variance were low (0.02-0.05), but the genetic standard deviations were high relative to the population average residual variance (25-60\%) (reviewed by Mulder et al. [30]).

When the aim is to change the mean and the variance of a trait simultaneously, e.g. by applying index selection, not only the genetic parameters but also the economic values for mean and variance of the trait need to be known. For most traits, economic values have been derived for their means, but not for their variances. Because the variance of a trait is a quadratic function of trait value, it will have a non-zero economic value if the profit equation is non-linear.

The effects of selection strategies on responses in mean and variance have been investigated for mass selection [17,30], canalising selection using a quadratic index with phenotypic information of progeny [36,37], index selection using arbitrary weights to increase the mean and to decrease the variance with repeated measurements on the same animal [38], and for selection either on progeny mean or on within-family variance [30]. None of these studies, however, investigated prospects for changing simultaneously the mean and the variance by using a selection index with optimal weights. The framework developed by Mulder et al. [30] allows extension to a selection index to optimise responses in the mean and the variance.

The objective of this study was to investigate the effects of genetic parameters, breeding goals, the number of progeny per sire and breeding schemes, e.g. progeny and sib testing, when changing the mean and the variance of a trait by exploiting genetic heterogeneity of residual variance. Economic values for the mean and the variance are derived for situations with non-linear profit and these economic values are applied in index selection to study response to selection. 


\section{MATERIAL AND METHODS}

\subsection{Genetic model}

In this study, it is assumed that selection is for a single trait in the presence of genetic heterogeneity of residual variance. Both the mean and the residual variance are partly under genetic control according to the model [17]:

$$
P=\mu+A_{m}+E
$$

with $E \sim N\left(0, \sigma_{E}^{2}+A_{v}\right)$, where $P$ is the phenotype, $\mu$ and $\sigma_{E}^{2}$ are, respectively, the mean trait value and the mean residual variance of the population, $A_{m}$ and $A_{v}$ are, respectively, the breeding value for the level and the residual variance of the trait. It is assumed that $A_{m}$ and $A_{v}$ follow a multivariate normal distribution $N\left(\left(\begin{array}{l}0 \\ 0\end{array}\right), \mathbf{C} \otimes \mathbf{A}\right)$, where $\mathbf{A}$ is the additive genetic relationship matrix, $\mathbf{C}=\left[\begin{array}{ll}\sigma_{A_{m}}^{2} & \operatorname{cov}_{A_{m v}} \\ \operatorname{cov}_{A_{m v}} & \sigma_{A_{v}}^{2}\end{array}\right], \sigma_{A_{m}}^{2}$ and $\sigma_{A_{v}}^{2}$ are the additive genetic variances in $A_{v}$ and $A_{m}$, respectively, $\operatorname{cov}_{A_{m v}}=\operatorname{cov}\left(A_{m}, A_{v}\right)=r_{A} \sigma_{A_{m}} \sigma_{A_{v}}$, and $r_{A}$ is the additive genetic correlation between $A_{m}$ and $A_{v}$. The mean phenotypic variance of the population $\left(\sigma_{P}^{2}\right)$ is the sum of $\sigma_{A_{m}}^{2}$ and $\sigma_{E}^{2}$. The mean phenotypic variance is independent of $A_{v}$ because $E\left(A_{v}\right)=0$. In contrast, the variance of a particular genotype, say $k$, depends on $A_{v_{k}}$ and is equal to $\sigma_{P_{k}}^{2}=\sigma_{E}^{2}+A_{v_{k}}$. In this study, the residual variance is equal to the environmental variance, assuming no other genetic or environmental complexities and using an animal model in genetic evaluation. The distribution of $P$ is approximately normal, but is slightly leptokurtic (coefficient of kurtosis $=3 \sigma_{A_{v}}^{2} / \sigma_{P}^{4}$ ) and, when $r_{A} \neq 0$, also slightly skewed (coefficient of skewness $=3 \operatorname{cov}_{A_{m v}} / \sigma_{P}^{3}$ ).

\subsection{Breeding schemes}

Breeding schemes are based on either sib testing or progeny testing. Sib testing is considered as the basis because it is most commonly applied in pig and poultry improvement, in which uniformity of animals is likely to be of most interest $[11,14]$. Progeny testing is considered as an alternative with the advantage of a higher accuracy of selection, which is (partly) offset by a longer generation interval.

Selection is for one trait and the breeding goal comprises both its mean and variance:

$$
H=v_{A_{m}} A_{m}+v_{A_{v}} A_{v}=\mathbf{v}^{\prime} \mathbf{a}
$$


where $H$ is the aggregate genotype, $v_{A_{m}}$ and $v_{A_{v}}$ are respectively the economic values for $A_{m}$ and $A_{v}, \mathbf{v}^{\prime}=\left[v_{A_{m}} v_{A_{v}}\right]$ and $\mathbf{a}^{\prime}=\left[A_{m} A_{v}\right]$. The trait is measured in both sexes before selection (e.g. body weight). The available phenotypic information is the following: own phenotype $P$, own phenotype squared $P^{2}$, mean phenotype of half-sibs $\bar{P}$, the square of the mean phenotype of halfsibs $(\bar{P})^{2}$ and the within-family variance of half-sibs varW. It is assumed that half-sib groups consist of 50 individuals with one progeny per dam to keep the selection index relatively simple, although in pigs and poultry dams have multiple progeny. The half-sib groups consist of males and females, assuming correction has been made for any sex effect on the mean and sexes do not differ in residual variance. Sires are either sib tested or progeny tested; dams are always sib tested. Generations are discrete. In each generation, $20 \%$ of the dams and $5 \%$ of the sires are selected by truncation on an index $I$ :

$$
I=\mathbf{b}^{\prime} \mathbf{x}
$$

where $\mathbf{b}=\mathbf{P}^{\mathbf{- 1}} \mathbf{G v}, \mathbf{x}$ is the vector with phenotypic information, expressed as deviations from the expectations, $\mathbf{P}=\operatorname{cov}(\mathbf{x}, \mathbf{x})$ and $\mathbf{G}=\operatorname{cov}(\mathbf{x}, \mathbf{a})$. Details of the $\mathbf{P}$ and $\mathbf{G}$ matrices are in Appendix A.

\subsection{Economic values for common cases with non-linear profit}

In this section, economic values for the mean and variance are derived for some standardised situations with non-linear profit. A non-zero economic value for variance implies that profit is non-linear in phenotype, because the variance of a trait is a quadratic function of its value. The clearest example of non-linear profit is for traits with an intermediate optimum e.g. [10,19].

\subsubsection{Quadratic profit}

Traits may have a quadratic profit equation with the maximum profit at an intermediate optimum value. An example is days open in dairy cattle [13]. A quadratic profit equation for an individual animal with phenotype $P$ is the following:

$$
M=r_{1}(P-O)^{2}+r_{2}
$$

where $M$ is the profit of an animal, $r_{1}$ and $r_{2}$ are the coefficients of the profit equation with $r_{1}$ describing the curvature $\left(r_{1}<0\right)$ and $r_{2}$ the profit at the 
optimum value, $O$ of the trait. The average profit $(\bar{M})$ of the population is the following:

$$
\bar{M}=\int_{-\infty}^{\infty} M f(P) \mathrm{d} P=r_{1} \mu^{2}-2 r_{1} \mu O+r_{1} O^{2}+r_{2}+r_{1} \sigma_{P}^{2}
$$

where $f(P)$ is the probability density function of a normal distribution. The economic values are given by the first derivatives of equation (5):

$$
\begin{gathered}
v_{A_{m}}=\frac{\mathrm{d} \bar{M}}{\mathrm{~d} \mu}=2 r_{1}(\mu-O), \\
v_{A_{v}}=\frac{\mathrm{d} \bar{M}}{\mathrm{~d} \sigma_{P}^{2}}=r_{1} .
\end{gathered}
$$

The ratio of $v_{A_{m}}$ to $v_{A_{v}}$ depends solely on the location of the population mean relative to the optimum trait value (see App. B). The relative weight on $A_{m}$ decreases as the population mean approaches the optimum.

\subsubsection{Differential profit based on thresholds}

In some practical cases, profit is not a continuous function of phenotype, but is discontinuous with differential revenues according to thresholds. Examples are $\mathrm{pH}$ in pork [19] or egg weight in poultry [34]. Assume that animals with a phenotype between the lower threshold $\left(T_{l}\right)$ and higher threshold $\left(T_{u}\right)$ have a profit $M=1$ and those outside these thresholds have a profit $M=0$ (see Fig. 1 for a schematic representation). The average profit of the population is:

$\bar{M}=M_{P<T_{l}} \int_{-\infty}^{T_{l}} f(P) \mathrm{d} P+M_{T_{l}<P<T_{u}} \int_{T_{l}}^{T_{u}} f(P) \mathrm{d} P+M_{P>T_{u}} \int_{T_{u}}^{\infty} f(P) \mathrm{d} P=\int_{T_{l}}^{T_{u}} f(P) \mathrm{d} P$.

The economic values are:

$$
\begin{gathered}
v_{A_{m}}=\frac{\mathrm{d} \bar{M}}{\mathrm{~d} \mu}=\frac{\mathrm{d} \bar{M}}{\mathrm{~d} t} \frac{\mathrm{d} t}{\mathrm{~d} \mu}=\frac{z_{l}-z_{u}}{\sigma_{P}}, \\
v_{A_{v}}=\frac{\mathrm{d} \bar{M}}{\mathrm{~d} \sigma_{P}^{2}}=\frac{\mathrm{d} \bar{M}}{\mathrm{~d} t} \frac{\mathrm{d} t}{\mathrm{~d} \sigma_{P}^{2}}=\frac{\frac{1}{2}\left(z_{l} t_{l}-z_{u} t_{u}\right)}{\sigma_{P}^{2}},
\end{gathered}
$$

where $z_{l}$ and $z_{u}$ are, respectively, the ordinate of the standard normal distribution at the standardised lower and upper thresholds $t_{l}=\left(T_{l}-\mu\right) / \sigma_{P}$ and 


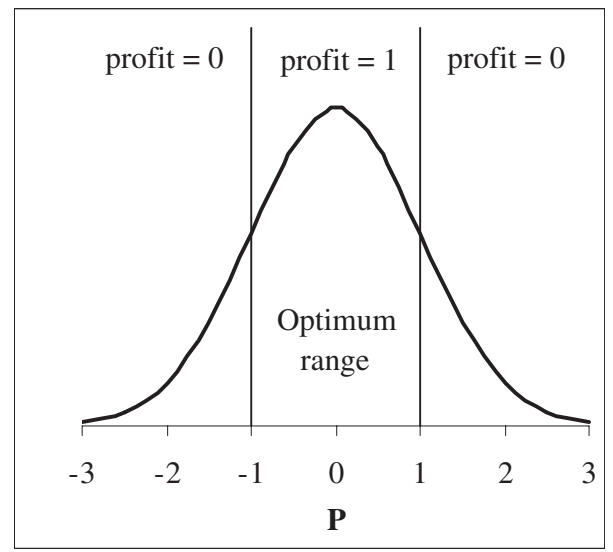

Figure 1. Schematic representation when profit is based on two thresholds $\left(T_{l}=-1\right.$, $T_{u}=1$ ) with optimum profit between both thresholds when the trait is normally distributed $(N(0,1)$; population mean $=$ optimum $=0)$.

$t_{u}=\left(T_{u}-\mu\right) / \sigma_{P}$. Equation (8a) is in agreement with previous research on economic values for optimum traits $[19,40]$, whereas $(8 b)$ is new. When the population mean is at the optimum $(\mu=O), v_{A_{m}}=0$ and $v_{A_{v}}<0$. The ratio of the absolute economic values $v_{A_{m}}$ and $v_{A_{v}}$ is determined mainly by the location of the population mean relative to both thresholds, but is also affected by $\sigma_{P}^{2}$. For determining the effect of economic values on genetic gain, however, the relative emphasis on the traits (e.g. $\left|\frac{v_{A_{v}} \sigma_{A_{v}}}{v_{A_{v}} \sigma_{A_{v}}+v_{A_{m}} \sigma_{A_{m}}}\right|$ ), is more relevant. Appendix B shows that the relative emphases on $A_{m}$ and $A_{v}$ are solely determined by the standardised deviation of the population mean from the optimum. This is also the case when economic values for optimum traits are based on quadratic profit.

Derivation of economic values can easily be extended to situations with several thresholds, as is shown for economic values for the mean of traits, e.g. calving ease in dairy cattle and meat quality in pigs $[2,9,40]$. A special case is one threshold, in which the terms relating to the second threshold in equations (8a) and (8b) can be omitted. An example is the avoidance of poor animal performance that may reduce consumer acceptance of the production system, so an objective may be to reduce the proportion of animals below a certain threshold [22].

\subsection{Prediction of genetic gain}

Genetic gain after one generation of selection was calculated deterministically using the classical selection index theory [15]. Most elements in the $\mathbf{P}$ 
and $\mathbf{G}$ matrices were derived by Mulder et al. [30]; the others are derived in Appendix A. Genetic gain was calculated per unit of time to account for the longer generation interval of sires with progeny testing, where one unit of time was equal to the generation interval of sib testing [29]. Genetic gain per unit of time for trait $j\left(A_{m}, A_{v}, H\right)$ was $\Delta G_{j}=\frac{R_{S, j}+R_{D, j}}{L_{S}+L_{D}}$, where $R_{S, j}$ and $R_{D, j}$ are the genetic selection differentials and $L_{S}$ and $L_{D}$ are the relative generation intervals for sires and dams, respectively. Genetic selection differentials for $A_{m}$ and $A_{v}$ were calculated as $R_{j}=\frac{i \mathbf{b}^{\prime} \mathbf{g}_{j}}{\sigma_{I}}$, where $i$ is the selection intensity, $\mathbf{g}_{j}$ is the column of $\mathbf{G}$ corresponding to $A_{m}$ or $A_{v}$, and $\sigma_{I}=\sqrt{\mathbf{b}^{\prime} \mathbf{P b}}$ is the standard deviation of the index. Genetic selection differentials of the aggregate genotype were calculated as $R_{H}=v_{A_{m}} R_{A_{m}}+v_{A_{v}} R_{A_{v}}$.

Gametic phase disequilibrium due to selection [5] was ignored. Although Hill and Zhang [17] developed prediction equations to account for gametic phase disequilibrium with mass selection, such equations have not yet been developed for index selection in the presence of genetic heterogeneity of residual variance. Selection intensities were calculated assuming an infinite population of selection candidates without correction for correlated index values among relatives $[16,27,31]$, because these corrections would have less effect on genetic gain than gametic phase disequilibrium, which was already ignored.

To check the quality of the predictions of the selection index equations for one generation of selection, predicted selection responses were compared with realised selection responses obtained from Monte Carlo simulation (see App. C). Prediction errors (Tab. A.I) were small to moderate, but sufficiently small to justify using selection index equations in this exploratory study.

\subsection{Parameter values and common cases with non-linear profit}

Parameter values are listed in Table I. The heritability of the mean $\left(h_{m}^{2}=\right.$ $\left.\sigma_{A_{m}}^{2} / \sigma_{P}^{2}\right)$ was assumed to be 0.3 ; the phenotypic variance was assumed to be 1.0. The genetic variance in residual variance $\sigma_{A_{v}}^{2}$ was varied between 0.01 and 0.10 , corresponding to the range of heritabilities of residual variance $\left(h_{v}^{2}=\sigma_{A_{v}}^{2} /\left(2 \sigma_{P}^{4}+3 \sigma_{A_{v}}^{2}\right)\right)$ observed in the literature (see [30] for derivation and review). The additive genetic correlation $\left(r_{A}\right)$ between $A_{m}$ and $A_{v}$ was varied between -0.5 and 0.5 , corresponding to the range in the literature for the analysis of body weight of snails, body weight of broilers and litter size of pigs $[33,35,38]$. Economic values $v_{A_{m}}$ and $v_{A_{v}}$ were varied and arbitrary values were initially used. In most species, the generation interval for progeny testing is at least 1.6 times that for sib testing e.g. $[25,26]$. Therefore, the relative generation interval of sib testing was set to 1.0 and that of progeny tested 
Table I. Parameter values used in the basic situation and in alternative situations.

\begin{tabular}{|c|c|c|}
\hline \multirow[t]{2}{*}{ Parameter } & \multicolumn{2}{|c|}{ Parameter values } \\
\hline & Basic & Alternative \\
\hline$\sigma_{4}^{2}$ & 0.3 & $0.1,0.6$ \\
\hline$\sigma_{P}^{A_{m}}$ & 1.0 & - \\
\hline$\sigma_{A}^{2}$ & 0.05 & $0.01,0.10$ \\
\hline$r_{A}^{A_{v}}$ & 0 & $-0.5,0.5$ \\
\hline$v_{A_{m}}$ & 1 & variable \\
\hline$v_{A_{m}}$ & -1 & variable \\
\hline Number of half-sib progeny & 50 & $20,50,100,200$ \\
\hline Selected proportion sires & 0.05 & - \\
\hline Selected proportion dams & 0.20 & - \\
\hline
\end{tabular}

sires was varied between 1.4 and 2 [29]. Responses to selection were predicted after one generation of selection, except for the cases with non-linear profit (see Sect. 2.5.1).

\subsubsection{Non-linear profit}

Sib testing schemes were simulated with three types of non-linear profit: quadratic profit $\left(r_{1}=-1, r_{2}=2\right.$ and $O=0$ ), and differential profit based on one threshold $\left(T_{l}=-1\right)$ or two thresholds $\left(T_{l}=-1, T_{u}=1, O=0\right)$. The initial population mean was $-2\left(=-2 \sigma_{P}\right)$. Five generations of selection were simulated with updating of economic values (Eqs. 6 and 8) and index weights to changes in mean and phenotypic variance. The elements of $\mathbf{P}$ were not, however, updated for changes in $\sigma_{E}^{2}$, i.e. ignoring changes in $h_{m}^{2}$ and $h_{v}^{2}$. To avoid oscillations around the optimum when the mean of the trait was close to it for models of quadratic profit or differential profit based on two thresholds ( $<\Delta A_{m}$ in previous generation), the economic value $v_{A_{m}}$ was derived iteratively to obtain the desired gain in $A_{m}$ to reach and stay in the optimum, similar to a desired gains approach e.g. [3].

\section{RESULTS}

\subsection{Effects of parameters and breeding scheme}

\subsubsection{Genetic variances $\sigma_{A_{m}}^{2}$ and $\sigma_{A_{v}}^{2}$}

Table II shows genetic gain in $A_{m}, A_{v}$ and the effect on the residual variance after one generation of selection in a sib testing scheme $\left(\sigma_{E, 1}^{2}\right)$ for different 
Table II. Genetic gain ${ }^{\mathrm{a}}$ after one generation of index selection in sib testing schemes for different values of $\sigma_{A_{m}}^{2}$ and $\sigma_{A_{v}}^{2}$ for an arbitrary breeding goal $\left(v_{A_{m}}=1, v_{A_{v}}=-1\right)^{\mathrm{b}}$.

\begin{tabular}{|c|c|c|c|c|c|}
\hline \multicolumn{2}{|c|}{ Genetic parameters } & \multicolumn{2}{|c|}{ Genetic gain } & \multicolumn{2}{|c|}{ Residual variance } \\
\hline$\overline{\sigma_{A_{m}}^{2}}$ & $\overline{\sigma_{A_{v}}^{2}}$ & $\Delta A_{m}$ & $\Delta A_{v}$ & $\sigma_{E, 0}^{2}$ & $\overline{\sigma_{E, 1}^{2}}$ \\
\hline \multirow[t]{3}{*}{0.10} & 0.01 & 0.253 & -0.002 & 0.900 & 0.898 \\
\hline & 0.05 & 0.234 & -0.043 & 0.900 & 0.857 \\
\hline & 0.10 & 0.202 & -0.118 & 0.900 & 0.782 \\
\hline \multirow[t]{3}{*}{0.30} & 0.01 & 0.603 & -0.001 & 0.700 & 0.699 \\
\hline & 0.05 & 0.593 & -0.020 & 0.700 & 0.680 \\
\hline & 0.10 & 0.573 & -0.062 & 0.700 & 0.638 \\
\hline \multirow[t]{3}{*}{0.60} & 0.01 & 1.074 & -0.001 & 0.400 & 0.399 \\
\hline & 0.05 & 1.068 & -0.013 & 0.400 & 0.387 \\
\hline & 0.10 & 1.055 & -0.038 & 0.400 & 0.362 \\
\hline
\end{tabular}

${ }^{\mathrm{a}}$ Equals genetic gain per time unit.

bParameters values: $\sigma_{P}^{2}=1, r_{A}=0$, number of progeny per sire $=50$, selected proportion sires $=0.05$, selected proportion dams $=0.20$.

${ }^{\mathrm{c}}$ Residual variance in generation $0\left(\sigma_{E, 0}^{2}\right)$ and in generation $1\left(\sigma_{E, 1}^{2}\right)$ after selection.

values of $\sigma_{A_{m}}^{2}$ and $\sigma_{A_{v}}^{2}$. Because the relative generation interval of sib testing was set to 1 , genetic gain per time unit was equal to genetic gain per generation. When $\sigma_{A_{m}}^{2}$ increases, $\Delta A_{m}$ increases substantially and $\Delta A_{v}$ decreases, whereas when $\sigma_{A_{v}}^{2}$ increases the opposite occurs but to a lesser extent. Both trends agree with the behaviour of a selection index, which puts most emphasis on the trait with the highest heritability and/or with the largest contribution to the genetic variance in the breeding goal. The decrease in residual variance is $0.25 \%-13 \%$ of the current residual variance. Simultaneous improvement of the mean and the variance of a trait with index selection in sib testing schemes thus requires a heritability of residual variance of at least 0.02 , and the reduction of phenotypic variance by selecting for reduced residual variance is the largest for traits with a low heritability of the mean.

\subsubsection{Genetic correlation $r_{A}$ and breeding goal}

Table III shows the effect of $r_{A}$ and breeding goals with arbitrary economic values on genetic gain after one generation of selection in a sib testing scheme. With a relatively low emphasis on $A_{v}\left(\mathbf{v}^{\prime}=[1-1]\right), \Delta A_{v}$ is mostly a correlated response to selection on the mean, as indicated by the similar $\Delta A_{v}$ with $\mathbf{v}^{\prime}=\left[\begin{array}{ll}1 & 0\end{array}\right]$. When increasing the emphasis on $A_{v}, \Delta A_{v}$ is in the direction of the economic value and $\Delta A_{m}$ is now more affected by $r_{A}$. With a breeding 
Table III. Genetic gain ${ }^{\mathrm{a}}$ after one generation of index selection in sib testing schemes for different breeding goals with arbitrary sets of economic values and $r_{A}{ }^{\mathrm{b}}$.

\begin{tabular}{|c|c|c|c|c|c|c|}
\hline \multicolumn{3}{|c|}{ Breeding goal } & \multirow[b]{2}{*}{$r_{A}$} & \multicolumn{2}{|c|}{ Genetic gain } & \multirow[b]{2}{*}{$\sigma_{E, 1}^{2}{ }^{\mathrm{c}}$} \\
\hline Description & $\overline{v_{A_{m}}}$ & $v_{A_{v}}$ & & $\Delta A_{m}$ & $\Delta A_{v}$ & \\
\hline \multirow[t]{3}{*}{ Only $A_{m}$} & 1 & 0 & -0.50 & 0.603 & -0.123 & 0.577 \\
\hline & & & 0.00 & 0.603 & 0.000 & 0.700 \\
\hline & & & 0.50 & 0.603 & 0.123 & 0.823 \\
\hline \multirow[t]{6}{*}{ Both $A_{m}$ and $A_{v}$} & 1 & -1 & -0.50 & 0.599 & -0.133 & 0.567 \\
\hline & & & 0.00 & 0.593 & -0.020 & 0.680 \\
\hline & & & 0.50 & 0.594 & 0.107 & 0.807 \\
\hline & 1 & -5 & -0.50 & 0.569 & -0.146 & 0.554 \\
\hline & & & 0.00 & 0.443 & -0.076 & 0.624 \\
\hline & & & 0.50 & -0.025 & -0.091 & 0.609 \\
\hline \multirow[t]{3}{*}{ Only $A_{v}$} & 0 & -1 & -0.50 & 0.495 & -0.151 & 0.549 \\
\hline & & & 0.00 & 0.000 & -0.111 & 0.589 \\
\hline & & & 0.50 & -0.495 & -0.151 & 0.549 \\
\hline
\end{tabular}

${ }^{\text {a }}$ Equals genetic gain per time unit.

${ }^{\mathrm{b}}$ Parameter values: $\sigma_{P}^{2}=1, \sigma_{A_{m}}^{2}=0.3, \sigma_{E, 0}^{2}=0.7, \sigma_{A_{v}}^{2}=0.05$, number of progeny per sire $=$ 50 , selected proportion sires $=0.05$, selected proportion dams $=0.20$.

${ }^{\mathrm{c}}$ Residual variance in generation $1\left(\sigma_{E, 1}^{2}\right)$ after selection.

goal $\mathbf{v}^{\prime}=[1-5]$, the current $\sigma_{E}^{2}$ decreases by $11-21 \%$ after one generation of selection at the expense of a lower genetic gain in the mean $\left(\Delta A_{m}\right)$. Thus relatively large changes in residual variance in the desired direction are possible if substantial emphasis is put on $A_{v}$ in the breeding goal.

\subsubsection{Number of half-sibs}

Table IV shows genetic gain after one generation of index selection as a function of the number of half-sibs per sire family for sib testing schemes for two breeding goals with arbitrary sets of economic values, $\mathbf{v}^{\prime}=\left[\begin{array}{ll}1-5\end{array}\right]$ and $\mathbf{v}^{\prime}=[1-1]$. For both goals, $\Delta A_{v}$ decreases when the number of halfsibs increases, especially for the former, while for the latter, $\Delta A_{m}$ is almost constant and the increase in $\Delta H$ is small. For the breeding goal $\mathbf{v}^{\prime}=[1-5]$, $\Delta A_{m}$ decreases when the number of half-sibs increases, because more emphasis is given to $A_{v}$ by the index. The increase in $\Delta H$ is large when the number of half-sibs increases. To achieve a substantial reduction of residual variance, the size of half-sibs groups should be at least 50 . 
Table IV. Genetic gain ${ }^{\mathrm{a}}$ in $A_{m}$ and $A_{v}$ and in the aggregate genotype after one generation of index selection as a function of the number of half-sib progeny per sire for sib testing schemes for two breeding goals with arbitrary sets of economic values ${ }^{\mathrm{b}}$.

\begin{tabular}{cllccc}
\hline \multicolumn{2}{c}{ Breeding goal } & & \multicolumn{3}{c}{ Genetic gain } \\
\hline$v_{A_{m}}$ & $v_{A_{v}}$ & Number of progeny & $\Delta A_{m}$ & $\Delta A_{v}$ & $\Delta H$ \\
\hline 1 & -1 & 20 & 0.578 & -0.013 & 0.591 \\
& & 50 & 0.593 & -0.020 & 0.613 \\
& & 100 & 0.598 & -0.029 & 0.627 \\
& & 200 & 0.599 & -0.038 & 0.637 \\
1 & -5 & 20 & 0.468 & -0.052 & 0.728 \\
& & 50 & 0.443 & -0.076 & 0.821 \\
& & 100 & 0.414 & -0.099 & 0.906 \\
& 200 & 0.385 & -0.121 & 0.992 \\
\hline
\end{tabular}

${ }^{\text {a }}$ Equals genetic gain per time unit.

b Parameter values: $\sigma_{P}^{2}=1, \sigma_{A_{m}}^{2}=0.3, \sigma_{A_{v}}^{2}=0.05, r_{A}=0$, selected proportion sires $=0.05$, selected proportion dams $=0.20$.

\subsubsection{Progeny testing versus sib testing}

Table V shows genetic gain per time unit for progeny testing schemes in comparison to sib testing schemes after one generation of selection for two arbitrary breeding goals as a function of the relative generation interval of progeny tested sires. In these situations, progeny testing schemes are superior for decreasing the residual variance $\left(\Delta A_{v}\right)$, but are inferior for $\Delta A_{m}$ unless the relative generation interval of progeny tested sires is short $(=1.4)$. Progeny testing schemes give higher $\Delta H$ than sib testing schemes with $v_{A_{v}}=-1$ only when the relative generation interval of progeny tested sires is short $(=1.4)$, whereas with $v_{A_{v}}=-5$, they do so unless the relative generation interval exceeds 1.6. Progeny testing schemes are, therefore, superior to sib testing schemes for decreasing residual variance, but provide lower genetic gain in the aggregate genotype when the relative generation interval of progeny testing is larger than 1.6 and when the breeding goal is mainly to change $A_{m}$.

\subsection{Common cases with non-linear profit}

\subsubsection{Quadratic profit}

Figures 2A, 2B, 2C and 2D show respectively the economic values for $A_{m}$ and $A_{v}$, mean, phenotypic variance and the weighted profit of the population as a function of generation number for different values of $r_{A}$ with index selection 
Table V. Genetic gain after one generation of index selection, expressed as gain per time unit, in $A_{m}$ and $A_{v}$ and in the aggregate genotype for progeny testing schemes in comparison to sib testing schemes for two breeding goals with arbitrary sets of economic values as a function of the relative generation interval of progeny tested sires $\left(L_{S}\right)^{\mathrm{a}}$.

\begin{tabular}{ccccccr}
\hline & \multicolumn{3}{c}{ Breeding goal } & \multicolumn{3}{c}{ Genetic gain } \\
\hline Breeding scheme & $v_{A_{m}}$ & $v_{A_{v}}$ & $L_{S}$ & $\Delta A_{m}$ & $\Delta A_{v}$ & $\Delta H_{r e l} \mathrm{~b}^{\mathrm{b}}$ \\
\hline Sib & 1 & -1 & 1.0 & 0.593 & -0.020 & 100.0 \\
Progeny & & & 1.4 & 0.611 & -0.028 & 104.2 \\
& & & 1.6 & 0.564 & -0.026 & 96.2 \\
& & & 1.8 & 0.524 & -0.024 & 89.3 \\
Sib & \multirow{2}{*}{1} & -5 & 1.0 & 0.489 & -0.022 & 83.4 \\
Progeny & & & 1.4 & 0.428 & -0.098 & 100.0 \\
& & & 1.6 & 0.395 & -0.090 & 102.9 \\
& & & 1.8 & 0.367 & -0.084 & 95.6 \\
& & & 2.0 & 0.342 & -0.078 & 89.2 \\
\hline
\end{tabular}

a Parameter values: $\sigma_{P}^{2}=1, \sigma_{A_{m}}^{2}=0.3, \sigma_{A_{v}}^{2}=0.05, r_{A}=0$, number of progeny per sire $=50$, selected proportion sires $=0.05$, selected proportion dams $=0.20$.

b Genetic gain in the aggregate genotype as a percentage relative to a sib testing scheme $\left(\Delta H / \Delta H_{s i b}\right) * 100 \%$.

for five generations in sib testing schemes for a quadratic profit equation. In the first three generations $v_{A_{m}}$ is much larger than $v_{A_{v}}$ in absolute terms (Fig. 2A). The population mean reaches the optimum in four generations and remains there in the fifth (Fig. 2B), as a consequence of a desired gains approach. Genetic gain in mean depends little on $r_{A}$ in the first three generations and the phenotypic variance changes mostly as a correlated response to selection on the mean (Fig. 2C). In generations 4 and 5, the phenotypic variance decreases for all values of $r_{A}$. Profit increases curvilinearly, as expected. The increase in profit is initially due mainly to increasing the mean to the optimum value (Fig. 2D), and the subsequent increase in profit due to decreasing phenotypic variance after generation 4 is relatively small. Therefore, for optimum traits with quadratic profit, it is most important to bring the mean to the optimum value and then to reduce the variance of the population when at the optimum.

\subsubsection{Differential profit based on one threshold}

Figure $3 \mathrm{~A}$ shows the general behaviour of equations (8a) and (8b) as a function of the population mean when profit is based on one threshold. 

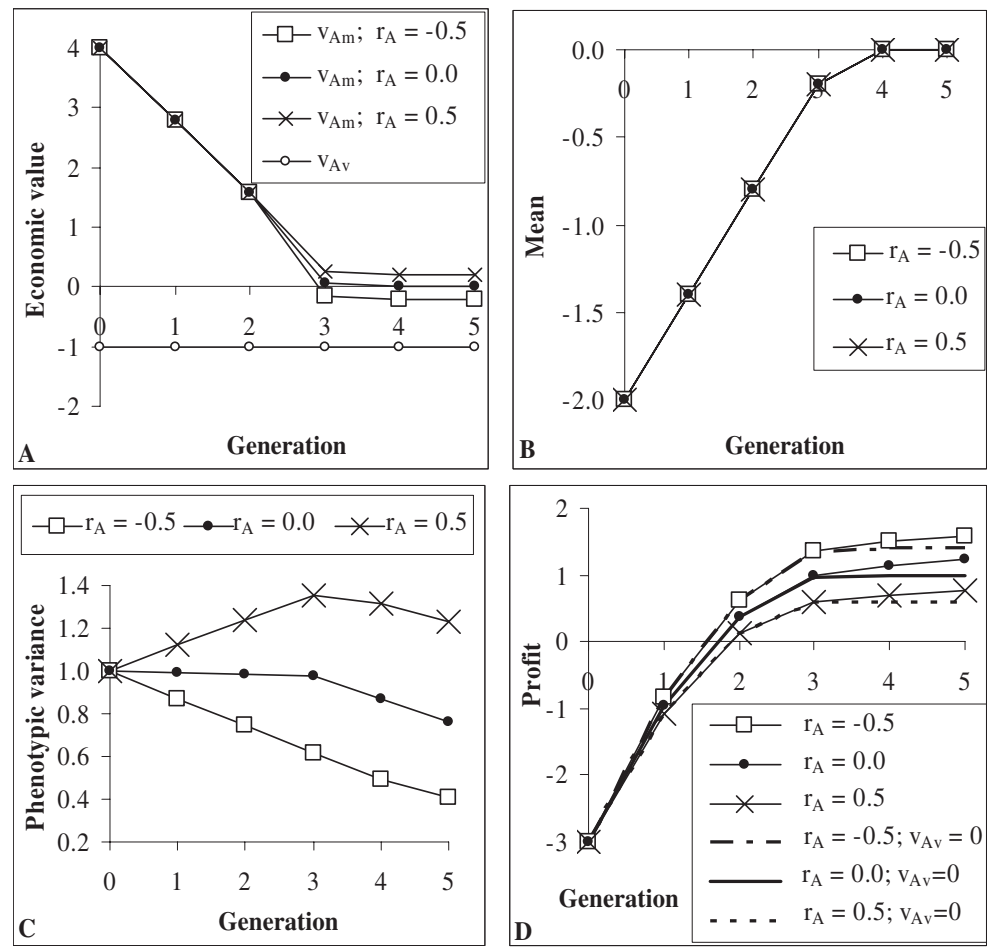

Figure 2. Economic values for $A_{m}\left(v_{A_{m}}\right)$ and $A_{v}\left(v_{A_{v}}\right)$ (panel A), mean (panel B), phenotypic variance (panel C) and profit (panel D) as a function of generation number with a quadratic profit equation $\left(M=-(P-0)^{2}+2\right)$ for different values of the genetic correlation $\left(r_{A}\right)$ with index selection for five generations of selection in sib testing schemes; panel $\mathrm{D}$ also shows the profit as a function of generation number when ignoring $A_{v}$ (lines without markers, $v_{A_{v}}=0$ ). Input parameters: $\sigma_{P}^{2}=1, \sigma_{A_{m}}^{2}=0.3, \sigma_{A_{v}}^{2}=0.05$, number of progeny per sire $=50$, selected proportion sires $=0.05$, selected proportion dams $=0.20$.

The economic value $v_{A_{m}}$ is maximum and $v_{A_{v}}$ is zero at the threshold, and animals below and above the threshold are equally frequent, such that the profit would increase substantially when the mean increases, but not when the variance changes. While $v_{A_{m}}$ is always positive, $v_{A_{v}}$ is positive (negative) when the population mean is lower (higher) than the threshold, because increasing (decreasing) the variance would increase the frequency of animals above the threshold.

Figures $4 \mathrm{~A}$ and $4 \mathrm{~B}$ show the population mean and the phenotypic variance as a function of generation number with index selection for five generations in sib testing schemes. When profit is based on one threshold, the population 

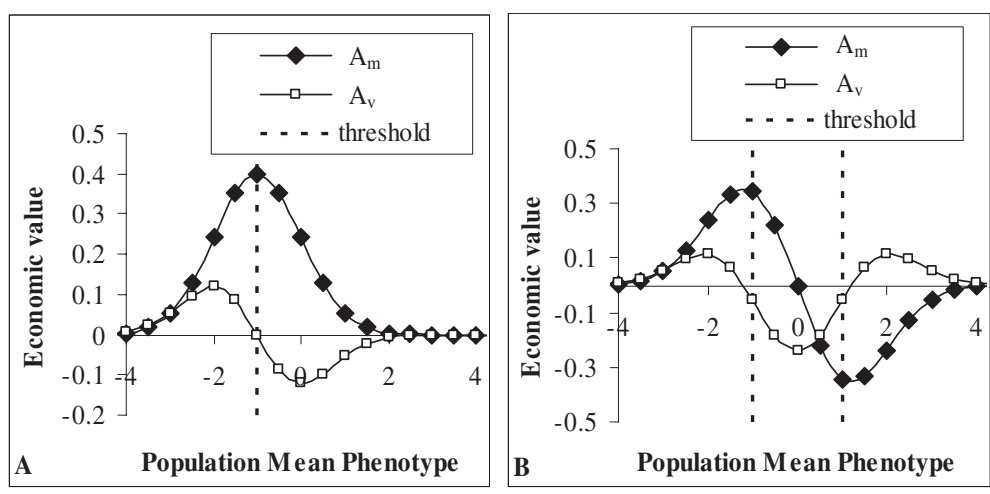

Figure 3. Economic values for $A_{m}$ and $A_{v}$ in the base generation as a function of the population mean for a normally distributed trait when profit is based on one threshold $\left(T_{l}=-1\right)\left(\right.$ panel A) or two thresholds $\left(T_{l}=-1, T_{u}=1, O=0\right)\left(\right.$ panel B) $\left(\sigma_{P}^{2}=1\right)$.
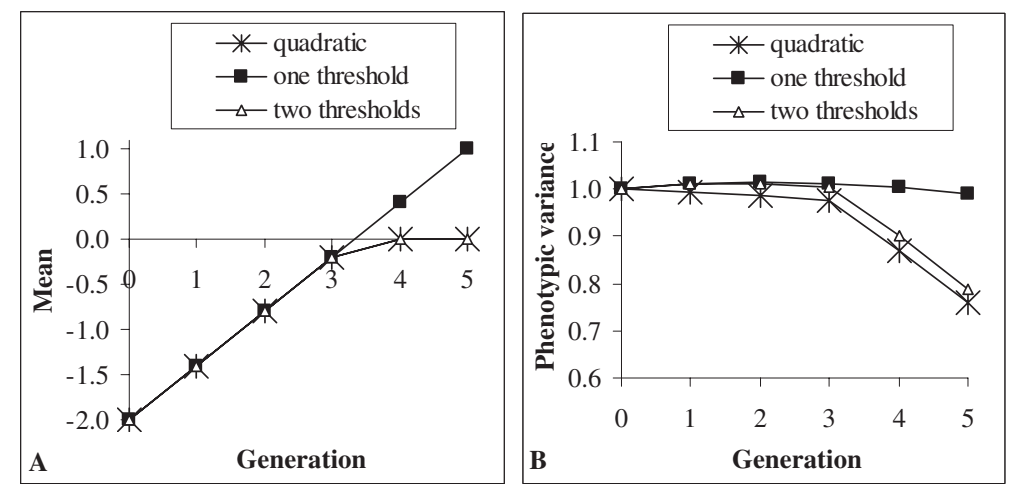

Figure 4. The mean (panel A) and phenotypic variance (panel B) as a function of generation number with index selection on non-linear profit for five generations of selection in sib testing schemes (quadratic $\left(r_{1}=-1, r_{2}=2\right.$ and $O=0$ ), one threshold $\left(T_{l}=-1\right)$ or two thresholds $\left(T_{l}=-1, T_{u}=1, O=0\right)$ ). Input parameters: $\sigma_{P}^{2}=1$, $\sigma_{A_{m}}^{2}=0.3, \sigma_{A_{v}}^{2}=0.05, r_{A}=0$, number of progeny per sire $=50$, selected proportion sires $=0.05$, selected proportion dams $=0.20$.

mean increases almost constantly with each generation. The phenotypic variance increases slightly in the first two generations and decreases slightly afterwards, because $v_{A_{v}}$ changes from positive to negative but is small relative to $v_{A_{m}}$. Thus with one threshold, most emphasis is on changing the mean and changing the variance is of minor importance. 


\subsubsection{Differential profit based on two thresholds}

When profit is based on two thresholds, the economic value of $A_{m}\left(v_{A_{m}}\right)$ is at a maximum or minimum close to both thresholds, is zero in the optimum $P=0$ and is positive (negative) when the population mean is lower (higher) than the optimum (Fig. 3B). When the population mean is outside both thresholds, $v_{A_{v}}$ is slightly positive because increasing the variance will increase the frequency of animals within the thresholds. When the population mean is within the thresholds, $v_{A_{v}}$ is negative because decreasing variance will increase the frequency of animals within the optimum range. The pattern of $v_{A_{m}}$ is similar to that observed by Hovenier et al. [19].

With continuous index selection, the mean increases up to the optimum (Fig. 4A) and the phenotypic variance increases for the first two generations and decreases afterwards at a substantial rate after generation 3 when the mean is (almost) in the optimum (Fig. 4B). Due to the decreased phenotypic variance, $74 \%$ of the animals in generation 5 are within the optimum range, whereas only $68 \%$ of the animals would have been so without selection for reduced phenotypic variance. The changes in the mean and phenotypic variance are similar to those for quadratic profit and, furthermore, also very similar for other threshold values (results not shown). As a generalisation, changes in the mean and variance of optimum traits do not seem to be very sensitive to the shape of the profit equation, and the selection index first drives the mean to reach the optimum and then targets a reduction in the phenotypic variance.

\section{DISCUSSION}

\subsection{Methodology and results}

In this study, possibilities for exploiting genetic heterogeneity of residual variance to change both the mean and the variance of a trait by index selection were explored. In general, for most traits the heritability of residual variance is low [30], in the range 0.02-0.05 [33,35-38], and also lower than the heritability of the mean. Consequently, breeding to change the mean and the variance simultaneously is similar to breeding for two traits, one with a moderate heritability and the other with a low heritability.

Inclusion of residual variance in the breeding goal is important only when the profit equation is non-linear; but, even so, in most situations the optimal index puts more selection pressure on the mean so that changes in residual variance are small. If the population mean is near an optimum, however, changes 
in residual variance can be large, over $10 \%$ of the current level, because it has a high genetic coefficient of variation $\sigma_{A_{v}} / \sigma_{E}^{2}$ [30].

In this study, gametic phase disequilibrium due to selection (the "Bulmer effect") [5] was ignored, because prediction equations have not yet been developed to accommodate this with index selection in the presence of genetic heterogeneity of residual variance. In general, accounting for the Bulmer effect would decrease selection responses as a consequence of a lower genetic variance at equilibrium, but the influence on ranking of breeding schemes is typically small [42]. More important perhaps, the Bulmer effect leads to changes in the genetic variance of the mean and as such obscures the responses in phenotypic variance obtained from changing the residual variance. Its effect is at most about 6-8\% of the phenotypic variance for an initial $h_{m}^{2}=0.3$ (based on formulae of Dekkers [8] comparing the equilibrium genetic variance with the genetic variance in the base generation). However, if the breeding goal has been to select on the mean for more than two or three generations, the genetic variance of the mean becomes rather stable and responses in phenotypic variance would be due almost entirely to responses in residual variance.

In this study, selection responses were predicted using a selection index framework in which, in principle, fixed effects are assumed to be known without error. In practice, fixed and random effects are simultaneously estimated using a mixed model BLUP analysis. There may be fixed effects both for the mean (e.g. herd effect) and the variance (e.g. heterogeneity of variance between herds). Heterogeneity of variance between herds is commonly found for milk production of dairy cattle [4], for example, and can be accounted for in standard breeding value estimation e.g. [28]. A model with genetically structured residual variance can be used to estimate simultaneously breeding values and fixed effects for mean and residual variance $[36,38]$. Disentangling heterogeneity of residual variance due to genotype from that due to the herd environment is, however, challenging and may require larger half-sib groups rather than those used in this study. The results presented here should therefore be interpreted as using an effective number of half-sibs that is lower than the actual number.

There is some evidence that heterozygotes tend to have a smaller residual variance than homozygotes [23,24,32], while a few studies have reported the opposite (Lynch and Walsh [23]). If heterozygotes have a smaller residual variance, then selection for reduced variance would favour heterozygotes. Furthermore, inbreeding reduces the Mendelian sampling variance among progeny, such that selection for reduced variance among progeny would favour more inbred parents. Both aspects reduce genetic variance of the mean amongst 
selected individuals, which would be an unfavourable consequence while genetic improvement of the mean is still important. The effect of inbreeding level of a parent on Mendelian sampling variance in its progeny can be eliminated, however, by adjusting the within-family variance for the inbreeding level of the parents. Furthermore, the effect of a selective advantage of heterozygotes on genotype frequencies is negligible for the infinitesimal model. Although selection experiments in Drosophila and Tribolium have indeed shown that selection for reduced phenotypic variance decreases both the residual and the genetic variances $[6,21]$, it is not known whether the latter is due to a build up of gametic phase disequilibrium or breakdown of infinitesimal model assumptions.

\subsection{Exploiting genetic heterogeneity of residual variance in breeding programmes}

When there is genetic variation in residual variance and the economic value of variance (per unit ${ }^{2}$ ) is at least of the same magnitude as the economic value of the mean (per unit), it can be worthwhile to exploit this genetic heterogeneity in breeding programmes. We consider in turn steps needed for implementation in practice: (1) estimation of breeding values for residual variance, (2) construction of a selection criterion, and (3) optimisation of the breeding programme.

As a first step, breeding values for mean and residual variance could be estimated by extending the mixed model framework $[36,38]$ and implementing this in software for routine genetic evaluation, which might be a challenge in itself. Since the heritability of residual variance is low, estimated breeding values (EBV) for residual variance would heavily rely on family information. Large family group sizes (e.g. 50-100 half-sib progeny) are necessary to estimate $E B V_{v}$ with sufficient accuracy [30].

Secondly, when EBV are based on a multivariate approach [36,38], a linear selection index, $I=v_{A_{m}} E B V_{m}+v_{A_{v}} E B V_{v}$, could be used as the selection criterion. A linear index with economic values derived as first derivatives is, however, not optimal with non-linear profit equations [12]. Assuming no genetic heterogeneity of residual variance, Goddard [12] concluded that the best linear index is better than a non-linear index. Formally, the proposed index is not linear, because the $E B V_{v}$ is based upon quadratic terms of phenotype ( $\overline{P^{2}}$ and $\operatorname{var} W$ ), and consequently his conclusion does not hold with genetic heterogeneity of residual variance. San Cristobal-Gaudy et al. [36] used a quadratic index as proposed by Wilton et al. [41] to breed for an optimum trait with 
genetic heterogeneity of residual variance. Their quadratic index consisted of the squared difference of the progeny mean from the optimum and the withinfamily variance with equal weights. These weights are not optimal and therefore too much emphasis is placed on $\overline{P^{2}}$, which contains very little information about $A_{v}$ and none about $A_{m}$. Hence, it is worse than a linearized selection index with updated economic values in each generation as used in this study (results not shown). Such a linearised index is therefore recommended for practical implementation.

Finally, the breeding programme may need to be optimised when including residual variance in the breeding goal and in the index. For example, our results show that progeny testing schemes are more efficient in reducing residual variance than sib testing schemes (Tab. V). Therefore, when reducing variance is a major goal, progeny testing schemes may be better than sib testing schemes even at the cost of a longer generation interval.

\section{CONCLUSIONS}

This study shows that it is possible to change the mean and the variance of traits simultaneously in livestock breeding programmes if there is genetic heterogeneity of residual variance. Economic values for mean and variance were derived for some standard non-linear profit equations, e.g. for traits with an intermediate optimum. Inclusion of residual variance in the breeding goal is of importance only when the profit equation is non-linear. Thus for a trait with an intermediate optimum, most economic gain is initially due to change in the mean, but selection pressure shifts gradually from the mean to the variance as the optimum is approached. Near the optimum, uniformity becomes the main goal, and so reduction of variance could further improve economic merit. Progeny testing schemes are predicted to give more rapid change in the residual variance than sib testing schemes, but at the cost of a lower genetic gain in the mean, mainly due to prolonged generation intervals. Predicted responses in residual variance after one generation of selection were large, in some cases when the number of progeny per sire was at least 50 , by more than $10 \%$ of the current residual variance.

\section{ACKNOWLEDGEMENTS}

H.M. thanks Johan van Arendonk, Bart Ducro and Roel Veerkamp for helpful comments on earlier versions of this article and Egbert Knol and Addie Vereijken for valuable discussions about the practical relevance of this 
research. We thank two anonymous reviewers and the editor, Erling Strandberg, for helpful comments on an earlier version of this article. W.G.H. thanks the Biotechnology and Biological Sciences Research Council for research support.

\section{REFERENCES}

[1] Barwick S.A., Henzell A.L., Assessing the value of improved marbling in beef breeding objectives and selection, Austr. J. Agric. Res. 50 (1999) 503-512.

[2] Bekman H., van Arendonk J.A.M., Derivation of economic values for veal, beef and milk production traits using profit equations, Livest. Prod. Sci. 34 (1993) $35-56$.

[3] Brascamp E.W., Selection indices with constraints, Anim. Breed. Abstr. 52 (1984) 645-654.

[4] Brotherstone S., Hill W.G., Heterogeneity of variance amongst herds for milk production, Anim. Prod. 42 (1986) 297-303.

[5] Bulmer M.G., The effect of selection on genetic variability, Am. Nat. 105 (1971) 201-211.

[6] Cardin S., Minvielle F., Selection on phenotypic variation of pupa weight in Tribolium castaneum, Can. J. Genet. Cytol. 28 (1986) 856-861.

[7] Clay J.S., Vinson W.E., White J.M., Heterogeneity of daughter variances of sires for milk yield, J. Dairy Sci. 62 (1979) 985-989.

[8] Dekkers J.C.M., Asymptotic response to selection on best linear unbiased predictors of breeding values, Anim. Prod. 54 (1992) 351-360.

[9] Dekkers J.C.M., Optimal breeding strategies for calving ease, J. Dairy Sci. 77 (1994) 3441-3453.

[10] Dekkers J.C.M., Birke P.V., Gibson J.P., Optimum linear selection indexes for multiple generation objectives with non-linear profit functions, Anim. Sci. 61 (1995) 165-175.

[11] Garnier J.P., Klont R., Plastow G., The potential impact of current animal research on the meat industry and consumer attitudes towards meat, Meat Sci. 63 (2003) 79-88.

[12] Goddard M.E., Selection indices for non-linear profit functions, Theor. Appl. Genet. 64 (1983) 339-344.

[13] Groen A.F., Meuwissen T.H.E., Vollema A.R., Brascamp E.W., A comparison of alternative index procedures for multiple generation selection on non-linear profit, Anim. Prod. 59 (1994) 1-9.

[14] Hammerstedt R.H., Symposium summary and challenges for the future, Poultry Sci. 78 (1999) 459-466.

[15] Hazel L.N., The genetic basis for constructing selection indexes, Genetics 28 (1943) 476-490.

[16] Hill W.G., Order statistics of correlated variables and implications in genetic selection programmes, Biometrics 32 (1976) 889-902. 
[17] Hill W.G., Zhang X.-S., Effects of phenotypic variability of directional selection arising through genetic differences in residual variability, Genet. Res. Camb. 83 (2004) 121-132.

[18] Hohenboken W.D., The manipulation of variation in quantitative traits: a review of possible genetic strategies, J. Anim. Sci. 60 (1985) 101-110.

[19] Hovenier R., Brascamp E.W., Kanis E., van der Werf J.H.J., Wassenberg A.P.A.M., Economic values of optimum traits: the example of meat quality in pigs, J. Anim. Sci. 71 (1993) 1429-1433.

[20] Kanis E., van Pelt M.L., Bonekamp P.R.T., Knol E.F., Is within-family variation in carcass weight of pigs heritable? Proc. 8th World Congress Genet. Appl. Livest. Prod., Belo Horizonte, Brazil, 2006, Communication 06-23.

[21] Kaufman P.K., Enfield F.D., Comstock R.E., Stabilizing selection for pupa weight in Tribolium castaneum, Genetics 87 (1977) 327-341.

[22] Kolmodin R., Bijma P., Response to mass selection when genotype by environment interaction is modelled as a linear reaction norm, Genet. Sel. Evol. 36 (2004) 435-454.

[23] Lynch M., Walsh B., Genetics and analysis of quantitative traits, Sinauer Associates, Inc. Publishers, Sunderland, Massachusetts, US, 1998.

[24] Mackay T.F.C., Lyman R.F., Drosophila bristles and the nature of quantitative genetic variation, Philo. Trans. R. Soc. B. 360 (2005) 1513-1527.

[25] Merks J.W.M., Genotype $\times$ environment interactions in pig breeding programmes, Ph.D. thesis, Landbouwuniversiteit Wageningen, Wageningen, The Netherlands, 1988.

[26] Meuwissen T.H.E., A deterministic model for the optimization of dairy cattle breeding based on BLUP breeding value estimates, Anim. Prod. 49 (1989) 193202.

[27] Meuwissen T.H.E., Reduction of selection differentials in finite populations with a nested full-half sib family structure, Biometrics 47 (1991) 195-203.

[28] Meuwissen T.H.E., De Jong G., Engel B., Joint estimation of breeding values and heterogeneous variances of large data files, J. Dairy Sci. 79 (1996) 310-316.

[29] Mulder H.A., Bijma P., Effects of genotype $\times$ environment interaction on genetic gain in breeding programs, J. Anim. Sci. 83 (2005) 49-61.

[30] Mulder H.A., Bijma P., Hill W.G., Prediction of breeding values and selection responses with genetic heterogeneity of environmental variance, Genetics 175 (2007) 1895-1910.

[31] Rawlings J.O., Order statistics for a special class of unequally correlated multinormal variates, Biometrics 32 (1976) 875-887.

[32] Robertson F.W., Reeve E.C.R., Heterozygosity, environmental variation and heterosis, Nature 170 (1952) 286.

[33] Ros M., Sorensen D., Waagepetersen R., Dupont-Nivet M., San Cristobal M., Bonnett J.C., Mallard J., Evidence for genetic control of adult weight plasticity in the snail Helix aspersa, Genetics 168 (2004) 2089-2097.

[34] Rose S.P., Principles of poultry science, CAB International, Wallingford, UK, 1996.

[35] Rowe S.J., White I.M.S., Avendano S., Hill W.G., Genetic heterogeneity of residual variance in broiler chickens, Genet. Sel. Evol. 38 (2006) 617-635. 
[36] San Cristobal-Gaudy M., Elsen J.M., Bodin L., Chevalet C., Prediction of the response to a selection for canalisation of a continuous trait in animal breeding, Genet. Sel. Evol. 30 (1998) 423-451.

[37] San Cristobal-Gaudy M., Bodin L., Elsen J.M., Chevalet C., Genetic components of litter size variability in sheep, Genet. Sel. Evol. 33 (2001) 249-271.

[38] Sorensen D., Waagepetersen R., Normal linear models with genetically structured residual variance heterogeneity: a case study, Genet. Res. Camb. 82 (2003) 207-222.

[39] van Vleck L.D., Variation of milk records within paternal-sib groups, J. Dairy Sci. 51 (1968) 1465-1470.

[40] Von Rohr P., Hofer A., Künzi N., Economic values for meat quality traits in pigs, J. Anim. Sci. 77 (1999) 2633-2640.

[41] Wilton J.W., Anthony Evans D., van Vleck L.D., Selection indices for quadratic models of total merit, Biometrics 24 (1968) 937-949.

[42] Wray N.R., Hill W.G., Asymptotic rates of response from index selection, Anim. Prod. 49 (1989) 217-227.

\section{APPENDIX A: THE P AND G MATRIX OF THE SELECTION INDEX}

The $\mathbf{P}$ and $\mathbf{G}$ matrices used in setting up the selection index are given here for one generation of selection. Derivations of most elements were given by Mulder et al. [30] and those for others follow.

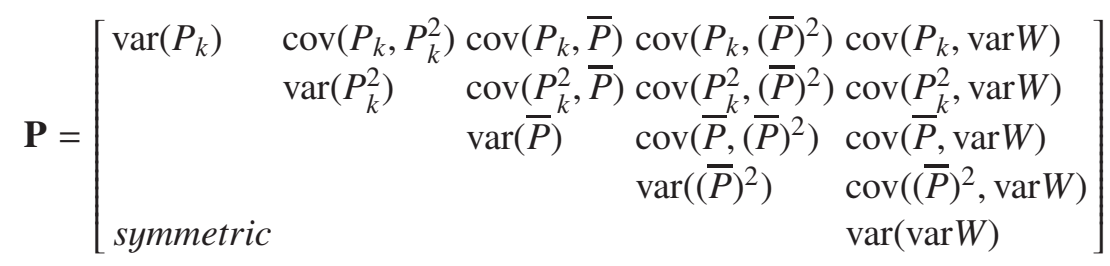

$$
\begin{aligned}
\operatorname{cov}\left(P_{k}, \bar{P}\right) & =n \operatorname{cov}\left(P_{k}, P_{l}\right) / n=a_{k} \sigma_{A_{m}}^{2} \\
\operatorname{cov}\left(P_{k}^{2}, \bar{P}\right) & =n \operatorname{cov}\left(P_{k}^{2}, P_{l}\right) / n=a_{k} \operatorname{cov}_{A_{m v}} \\
\operatorname{cov}\left(P_{k},(\bar{P})^{2}\right) & =\left[n \operatorname{cov}\left(P_{k}, P_{l}^{2}\right)+n(n-1) \operatorname{cov}\left(P_{k}, P_{l} P_{m}\right)\right] / n^{2}=a_{k} \operatorname{cov}_{A_{m v}} / n \\
\operatorname{cov}\left(P_{k}^{2},(\bar{P})^{2}\right) & =\left[n \operatorname{cov}\left(P_{k}^{2}, P_{l}^{2}\right)+n(n-1) \operatorname{cov}\left(P_{k}^{2}, P_{l} P_{m}\right)\right] / n^{2} \\
& =2 a_{k}^{2} \sigma_{A_{m}}^{4}+a_{k} \sigma_{A_{v}}^{2} / n \\
\operatorname{cov}\left(P_{k}, \operatorname{var} W\right) & =[n / n-1] \times\left[\operatorname{cov}\left(P_{k}, \overline{P^{2}}\right)-\operatorname{cov}\left(P_{k},(\bar{P})^{2}\right)\right]=a_{k} \operatorname{cov}_{A_{m v}} \\
\operatorname{cov}\left(P_{k}^{2}, \operatorname{var} W\right) & =[n / n-1] \times\left[\operatorname{cov}\left(P_{k}^{2}, \overline{P^{2}}\right)-\operatorname{cov}\left(P_{k}^{2},(\bar{P})^{2}\right)\right]=a_{k} \sigma_{A_{v}}^{2}
\end{aligned}
$$


and

$$
\mathbf{G}=\left[\begin{array}{ll}
\sigma_{A_{m}}^{2} & \operatorname{cov}_{A_{m v}} \\
\operatorname{cov}_{A_{m v}} & \sigma_{A_{v}}^{2} \\
a_{k} \sigma_{A_{m}}^{2} & a_{k} \operatorname{cov}_{A_{m v}} \\
a_{k} \operatorname{cov}_{A_{m v}} / n & a_{k} \sigma_{A_{v}}^{2} / n \\
a_{k} \operatorname{cov}_{A_{m v}} & a_{k} \sigma_{A_{v}}^{2}
\end{array}\right],
$$

where $a_{k}$ is the additive genetic relationship between animal $k$ and the group of half-sibs ( $a_{k}=0.25$ for sib testing; $a_{k}=0.5$ for progeny testing), $a_{w}$ is the additive genetic relationship among relatives within the group $\left(a_{w}=0.25\right.$ for half-sibs), and $l$ and $m$ are different half-sibs within the family.

\section{APPENDIX B: THE RELATIVE EMPHASIS ON VARIANCE FOR OPTIMUM TRAITS WITH QUADRATIC PROFIT AND DIFFERENTIAL PROFIT WITH TWO THRESHOLDS}

\section{Quadratic profit:}

The relative emphasis of $A_{v}$ in the breeding goal $\left(\operatorname{Rel}_{A_{v}}=\left|\frac{v_{A_{v}} \sigma_{A_{v}}}{v_{A_{v}} \sigma_{A_{v}}+v_{A_{m}} \sigma_{A_{m}}}\right|\right)$ with quadratic profit is $\operatorname{Rel}_{A_{v}}=\frac{G C V_{E}\left(1-h_{m}^{2}\right)}{G C V_{E}\left(1-h_{m}^{2}\right)+2 x h_{m}}$, where $x=\frac{|\mu-O|}{\sigma_{P}}$ and $G C V_{E}=$ $\sigma_{A_{v}} / \sigma_{E}^{2}$. $\operatorname{Rel}_{A_{v}}$ is therefore independent of $\sigma_{P}^{2}$ and completely determined by the distance of $\mu-O$ expressed in terms of $\sigma_{P}$.

Differential profit with two thresholds:

With differential profit based on two thresholds, $\operatorname{Rel}_{A_{v}}$ can be expressed as $\operatorname{Rel}_{A_{v}}=\frac{G C V_{E}\left(1-h_{m}^{2}\right)}{\operatorname{GCV}_{E}\left(1-h_{m}^{2}\right)+2 y h_{m}}$, where $y=\left|\frac{z_{l}-z_{u}}{z_{l} t_{l}-z_{u} t_{u}}\right|$, and is therefore independent of $\sigma_{P}^{2}$ and completely determined by the standardized distances of $\mu$ from $T_{l}$ and $T_{u}$. A first order Taylor series approximation of $y$ when $\mu=O$ is equal to $x$, showing the similarity between the quadratic model and the differential profit model with two thresholds when the population mean is close to the optimum.

\section{APPENDIX C: COMPARISON OF PREDICTED RESPONSES FROM SELECTION INDEX THEORY WITH REALISED RESPONSES FROM MONTE CARLO SIMULATION}

Monte Carlo simulation was used to evaluate the quality of the predictions as outlined in 2.4 for sib testing and progeny testing schemes after one generation of index selection with different breeding goals. Fifty replicates with 500000 sires each with 50 half-sib progeny were generated to mimic an infinite population. Dams were randomly mated to sires and each dam had 
Table A.1. Predicted genetic gain per time unit and prediction errors ${ }^{\mathrm{a}}$ for $A_{m}, A_{v}$ and the aggregate genotype $\left(\Delta A_{m}, \Delta A_{v}\right.$ and $\left.\Delta H\right)$ after one generation of selection with sib testing and progeny testing schemes for different breeding goals with arbitrary sets of economic values $\left(v_{A_{m}}=1 ; v_{A_{v}} \text { is varied }\right)^{\mathrm{b}}$.

\begin{tabular}{lcrrrrrr}
\hline & & \multicolumn{4}{c}{ Genetic gain (prediction error ${ }^{\mathrm{a}}$ ) } \\
\cline { 3 - 8 } Breeding scheme & $v_{A_{v}}$ & \multicolumn{2}{c}{$\Delta A_{m}$} & \multicolumn{2}{c}{$\Delta A_{v}$} & \multicolumn{1}{c}{$\Delta H$} \\
\hline Sib testing & 0 & 0.603 & $(0.003)$ & 0.000 & $(-0.031)$ & 0.603 & $(0.003)$ \\
& -1 & 0.593 & $(-0.001)$ & -0.020 & $(-0.027)$ & 0.613 & $(0.026)$ \\
Progeny testing & -5 & 0.444 & $(0.079)$ & -0.076 & $(0.004)$ & 0.822 & $(0.058)$ \\
& 0 & 0.581 & $(0.000)$ & 0.000 & $(-0.010)$ & 0.581 & $(0.000)$ \\
& -1 & 0.566 & $(-0.002)$ & -0.027 & $(-0.008)$ & 0.593 & $(0.006)$ \\
& -5 & 0.395 & $(0.036)$ & -0.092 & $(0.005)$ & 0.855 & $(0.011)$ \\
\hline
\end{tabular}

a Prediction errors between brackets: the deviation predicted - observed in Monte Carlo simulation averaged over all replicates.

b Parameter values: $\sigma_{P}^{2}=1, \sigma_{A_{m}}^{2}=0.3, \sigma_{A_{v}}^{2}=0.05, r_{A}=0$, number of progeny per sire $=50$, selected proportion sires $=0.05$, selected proportion dams $=0.20$, relative generation interval of progeny tested sires $L_{S}=1.6$.

one progeny. Input parameters were as the basic situation in Table I. The breeding values of sires and dams were randomly sampled normal variates with variances $\sigma_{A_{m}}^{2}$ and $\sigma_{A_{v}}^{2}$, respectively, assuming $r_{A}=0$. For each progeny, the Mendelian sampling terms were randomly sampled with variance $\frac{1}{2} \sigma_{A_{m}}^{2}$ and $\frac{1}{2} \sigma_{A_{v}}^{2}$, respectively, and then residuals were randomly sampled with variance $\sigma_{E}^{2}+A_{v}$. Sires and dams were selected by truncation on an index (Eq. 3). Sires were either sib tested or progeny tested; dams were always sib tested. Genetic selection differentials were calculated as the mean $A_{m}$ and $A_{v}$ of all selected sires and dams and averaged over replicates. Genetic gain per time unit was calculated, assuming a relative generation interval of progeny tested sires of 1.6. Prediction errors were small for $\Delta A_{m}$ when $v_{A_{v}}=0$ or $v_{A_{v}}=-1$, but larger when $v_{A_{v}}=-5$ (see Tab. A.I). For $\Delta A_{v}$ the opposite was observed: prediction errors were small when $v_{A_{v}}=-5$. Prediction errors were larger for sib testing schemes than for progeny testing schemes. 